\title{
Complex Capacitance Analysis on Leakage Current Appearing in Electric Double-layer Capacitor Carbon Electrode
}

\author{
Jong H. Jang, ${ }^{a}$ Songhun Yoon, ${ }^{a}$ Bok H. Ka, ${ }^{a}$ Yong-Ho Jung, ${ }^{b}$ and Seung M. Oh ${ }^{\mathrm{a}, *, \mathrm{z}}$ \\ ${ }^{a}$ School of Chemical and Biological Engineering and Research Center for Energy Conversion and \\ Storage, Seoul National University, Seoul 151-744, Korea \\ ${ }^{b}$ Ness Cap Corporation, 29-5, Suwon 442-380, Kyunggi-do, Korea
}

Complex capacitance analysis was done on the porous carbon electrode-electrolyte interface, where a minor leakage current is involved in addition to the dominant capacitor charging current. Based on the transmission line model, imaginary capacitance profiles $\left(C_{\text {im }} v s . \log f\right)$ were theoretically derived for a cylindrical pore and multiple pore systems of nonuniform pore geometry. The parallel $R C$ circuit was assumed for the interfacial impedance, where $R$ is the charge-transfer resistance for leakage current and $C$ the double-layer capacitance. The theoretical derivation illustrated that the resistive tail relevant to the leakage current appears in addition to the capacitive peak, which was in accordance with the experimental data taken on the porous carbon electrode. The electric double-layer capacitor (EDLC) parameters such as the extent of leakage current, total capacitance, and rate capability were visually estimated from the imaginary capacitance profiles. The more quantitative EDLC parameters were obtained by a nonlinear fitting to the experimental data.

(C) 2005 The Electrochemical Society. [DOI: 10.1149/1.1931469] All rights reserved.

Manuscript submitted May 24, 2004; revised manuscript received February 7, 2005. Available electronically June 10,2005

Electric double-layer capacitor (EDLC) utilizes the double layer formed at the electrode-electrolyte interface, where electric charges are stored on the electrode surface and ions of counter charge are arranged in the electrolyte side. The most demanding feature for EDLC electrodes is the ideally polarized behavior over a wide potential range. The practical EDLC electrodes, however, suffer from a self-discharge at the charged state that is caused by leakage currents. ${ }^{1-5}$ This nonideally polarized behavior should thus be minimized to improve the charge-discharge efficiency and reliability of commercial cells.

The leakage current appearing in EDLC electrodes can be analyzed using ac impedance spectroscopy. When impedance data are analyzed using the conventional Nyquist plot, the vertical line in the low-frequency region that is observed for ideally polarized electrodes becomes inclined with an increase in the leakage current. ${ }^{6-8}$ The extent of leakage current can thus be estimated from the degree of inclination. The problem here, however, is that the inclination is also observed even in ideally polarized electrodes when they have a nonuniform pore geometry that is common in most practical porous EDLC electrodes. ${ }^{9-12}$ In theory, the differentiation between the leakage current and nonuniform pore geometry for the cause of inclination is possible when the measurement is made at very low frequencies. In the extremely low-frequency region, ideally polarized electrodes with nonuniform pore geometry give a vertical line on the Nyquist plot, whereas the leakage current is visualized as a semicircle whose diameter reflects the charge-transfer resistance for leakage reaction. In practice, however, it is difficult to obtain data at such a low frequency due to an instrumental limitation and extremely long measuring time.

In this work, the complex capacitance analysis is done on the leakage current involved in porous carbon electrode. From the imaginary capacitance plot $\left(C_{\mathrm{im}} v s . \log f\right)$ that is derived from the experimental impedance data, the leakage current can be analyzed without the impedance data taken at extremely low frequencies $(<5 \mathrm{mHz})$. Moreover, as the leakage current appears as a resistive tail in the frequency region lower than that for the capacitive peak, whose broadness reflects the nonuniform pore geometry, the two problematic features can be analyzed separately. The imaginary capacitance is theoretically derived based on the transmission line model, and its usefulness in estimating EDLC parameters is verified by applying it to the porous carbon electrode.

\footnotetext{
* Electrochemical Society Active Member.

${ }^{\mathrm{z}}$ E-mail: seungoh@plaza.snu.ac.kr
}

\section{Theory}

An ideally polarized cylindrical pore.-Electrochemical characteristics of porous electrodes differ from those of planar ones because the electrolyte resistance in pores becomes larger with an increase in the distance from pore opening to the electrolyteelectrode interface made deep inside pores. The transmission line model (TLM) has been widely utilized to describe such a situation, where the electrolyte resistance in pores $\left(R_{\text {pore }}\right)$ and interfacial impedance $\left(Z_{\mathrm{i}, \mathrm{A}}\right)$ are taken as the basic circuit element (Fig. 1a). ${ }^{9-22}$ The overall impedance has been derived by de Levie as Eq. 1, assuming that the electrode resistance is negligible as compared to the electrolyte resistance in pores. ${ }^{13}$ Here, $Z_{\mathrm{i}, 0}$ and $R_{0}$ are the interfacial impedance and electrolyte resistance in a pore, respectively

$$
Z(f)=\sqrt{R_{0} Z_{\mathrm{i}, 0}(f)} \operatorname{coth}\left[\sqrt{\frac{R_{0}}{Z_{\mathrm{i}, 0}(f)}}\right]
$$

If the electrodes are ideally polarized, the interfacial impedance per unit surface area $\left(Z_{\mathrm{i}, \mathrm{A}}\right)$ can be simplified as a capacitor (Fig. 1b). For a single cylindrical pore (radius: $r$, length: $L$ ), the impedance and complex capacitance are derived as Eq. 3 and $4,{ }^{22}$

$$
\begin{gathered}
Z_{\mathrm{i}, 0}(f)=\frac{1}{j 2 \pi f C_{0}}, \quad R_{0}=\frac{L}{\kappa \pi r^{2}} \\
Z(f)=\frac{R_{0}}{\sqrt{j 2 \pi \tau_{1} f}} \operatorname{coth}\left[\sqrt{j 2 \pi \tau_{1} f}\right] \\
C(f)=\frac{C_{0}}{\sqrt{j 2 \pi \tau_{1} f}} \tanh \left[\sqrt{j 2 \pi \tau_{1} f}\right] \\
\tau_{1}=R_{0} C_{0}=2\left(\frac{C_{\mathrm{A}}}{\kappa}\right)\left(\frac{L^{2}}{r}\right)
\end{gathered}
$$

Here, the time constant $\left(\tau_{1}\right)$ is expressed as a combination of electrochemical parameters $\left(C_{\mathrm{A}}\right.$ : double-layer capacitance per unit area, $\kappa$ : electrolyte conductivity in pore) and pore geometry ( $r$ : pore radius, $L$ : pore length). $R_{0}$ and $C_{0}$ are the electrolyte resistance and total capacitance for a pore, respectively. Note that $\tau_{1}$ characterizes the rate capability of EDLC electrodes as the capacitor charging time becomes longer with an increase in $\tau_{1}$ value. By plotting the imaginary part of complex capacitance as a function of frequency $\left(C_{\text {im }} v s . \log f\right)$, a peak-shaped curve is obtained, from which the total capacitance $\left(C_{0}\right)$ and time constant $\left(\tau_{1}\right)$ for a single pore can be estimated from the integrated peak area $(A)$ and peak frequency $\left(f_{p}\right)$ as expressed in Eq. $6,^{22}$ 
(a)



(b)

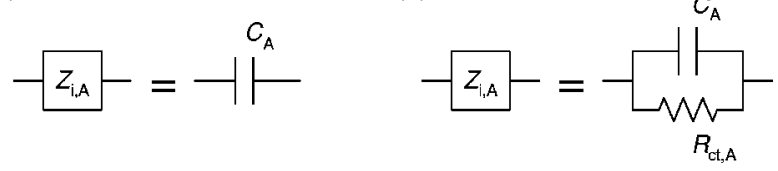

Figure 1. (a) The equivalent circuit for transmission line model that is composed of pore resistance $\left(R_{\text {pore }}\right)$ and interfacial impedance $\left(Z_{\mathrm{i}, \mathrm{A}}\right)$; (b) the equivalent circuit for interfacial impedance in the case of ideally polarized electrode; and (c) the equivalent circuit for nonideally polarized electrode. $R_{\mathrm{ct}, \mathrm{A}}$ is the charge transfer resistance for leakage reaction.

$$
A=0.682 C_{0}, \quad f_{p}=0.404 / \tau_{1}
$$

A nonideally polarized cylindrical pore.-When leakage current is involved in addition to capacitor charging current, the interfacial impedance of the electrode-electrolyte interface should be expressed as a parallel combination of capacitor $\left(C_{\mathrm{A}}\right)$ and resistance $\left(R_{\mathrm{ct}, \mathrm{A}}\right)$ (Fig. 1c). The interfacial impedance is

$$
\begin{gathered}
Z_{\mathrm{i}, 0}(f)=\frac{1}{j 2 \pi f C_{0}} \frac{1}{1+\left(j 2 \pi \tau_{2} f\right)^{-1}} \\
\tau_{2}=R_{\mathrm{ct}} C_{0}
\end{gathered}
$$

Here, another time constant $\left(\tau_{2}\right)$ is defined as the product of total capacitance $\left(C_{0}\right)$ and charge-transfer resistance $\left(R_{\mathrm{ct}}\right)$ for leakage current; thereby, a larger $\tau_{2}$ reflects a smaller leakage current. By inserting Eq. 7 into Eq. 1, the overall impedance and complex capacitance for a cylindrical pore are derived as follows

$$
\begin{aligned}
& Z(f)=R_{0} \frac{1}{\sqrt{j 2 \pi \tau_{1} f}} \frac{1}{\sqrt{1+\frac{1}{j 2 \pi \tau_{2} f}}} \operatorname{coth}\left[\sqrt{j 2 \pi \tau_{1} f} \sqrt{1+\frac{1}{j 2 \pi \tau_{2} f}}\right] \\
& C(f)=C_{0} \frac{1}{\sqrt{j 2 \pi \tau_{1} f}} \sqrt{1+\frac{1}{j 2 \pi \tau_{2} f}} \tanh \left[\sqrt{j 2 \pi \tau_{1} f} \sqrt{1+\frac{1}{j 2 \pi \tau_{2} f}}\right]
\end{aligned}
$$

When the imaginary part of complex capacitance is plotted against frequency $\left(C_{\text {im }} v s . \log f\right)$, the resistive tail that originates from the charge-transfer reaction for leakage current is observed at the lower frequency region in addition to the capacitive peak that is associated with the capacitor charging (Fig. 2). When $\tau_{2}$ is sufficiently larger than $\tau_{1}\left(\right.$ e.g., $\left.\tau_{2} / \tau_{1}>10^{5}\right)$, that is, the leakage current is negligible, the resistive tail and capacitive peak are completely separated (Fig. 2a). As $\tau_{2}$ approaches $\tau_{1}$, however, the resistive tail gradually shifts to the higher frequency direction to overlap with the capacitive peak. The capacitive peak in nonideally polarized electrodes shows the same property as that for ideally polarized ones: The area $(A)$ and peak position $\left(f_{\mathrm{p}}\right)$ of capacitive peak are proportional to the total capacitance $\left(C_{0}\right)$ and the inverse of $\tau_{1}$ (Eq. 6) as simulated in Fig. 2b and c.

Nonideally polarized multiple pore systems.-As porous carbons used in practical EDLC electrodes contain a large number of nonuniform pores that differ in both radius and length, $\tau_{1}$ should
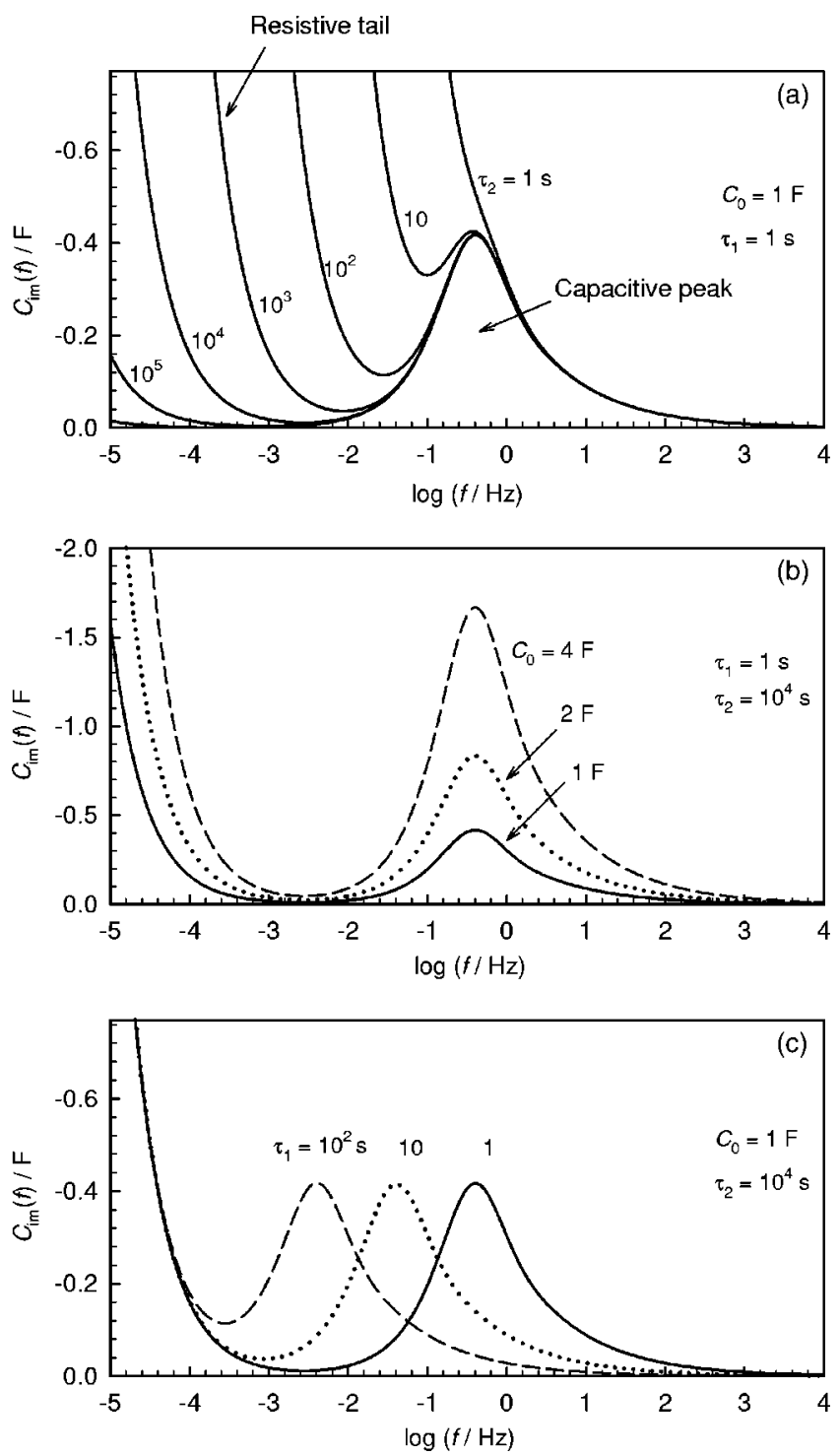

Figure 2. The simulated imaginary capacitance profiles $\left(C_{\mathrm{im}} v s . \log f\right)$ for a cylindrical pore. (a) The evolution of resistive tail as a function of $\tau_{2}\left(C_{0}\right.$ and $\tau_{1}$ are fixed at $1 \mathrm{~F}$ and $1 \mathrm{~s}$, respectively); (b) the relationship between total capacitance $\left(C_{0}\right)$ and peak area $\left(\tau_{1}\right.$ and $\tau_{2}$ are fixed at 1 and $10^{4} \mathrm{~s}$, respectively); and (c) the shift of capacitive peak as a function of $\tau_{1}\left(C_{0}\right.$ and $\tau_{2}$ are fixed at $1 \mathrm{~F}$ and $10^{4} \mathrm{~s}$, respectively)

have a certain distribution rather than a single value (Eq. 5). Considering this, the imaginary capacitance for multipore systems is derived as follows

$$
C_{\mathrm{im}}(f)=\int_{0}^{\infty} C_{\mathrm{TLM}-(\mathrm{RC})}^{0}\left(f, \tau_{1}, \tau_{2}\right) C_{\mathrm{tot}} p\left(\tau_{1}\right) d \log \tau_{1}
$$

Here, $C_{\mathrm{TLM}-(\mathrm{RC})}^{0}\left(f, \tau_{1}, \tau_{2}\right)$ is a transfer function for nonideally polarized interface, which is defined as Eq. 12. $C_{\text {tot }}$ and $p\left(\tau_{1}\right)$ are the total capacitance of multiple pore systems and logarithmic distribution function for $\tau_{1}$ (Eq. 13), respectively

$$
\begin{aligned}
& C_{\mathrm{TLM}-(\mathrm{RC})}^{0}\left(f, \tau_{1}, \tau_{2}\right) \\
& \quad=\operatorname{Im}\left[\frac{1}{\sqrt{j 2 \pi \tau_{1} f}} \sqrt{1+\frac{1}{j 2 \pi \tau_{2} f}} \tanh \left[\sqrt{j 2 \pi \tau_{1} f} \sqrt{1+\frac{1}{j 2 \pi \tau_{2} f}}\right]\right]
\end{aligned}
$$



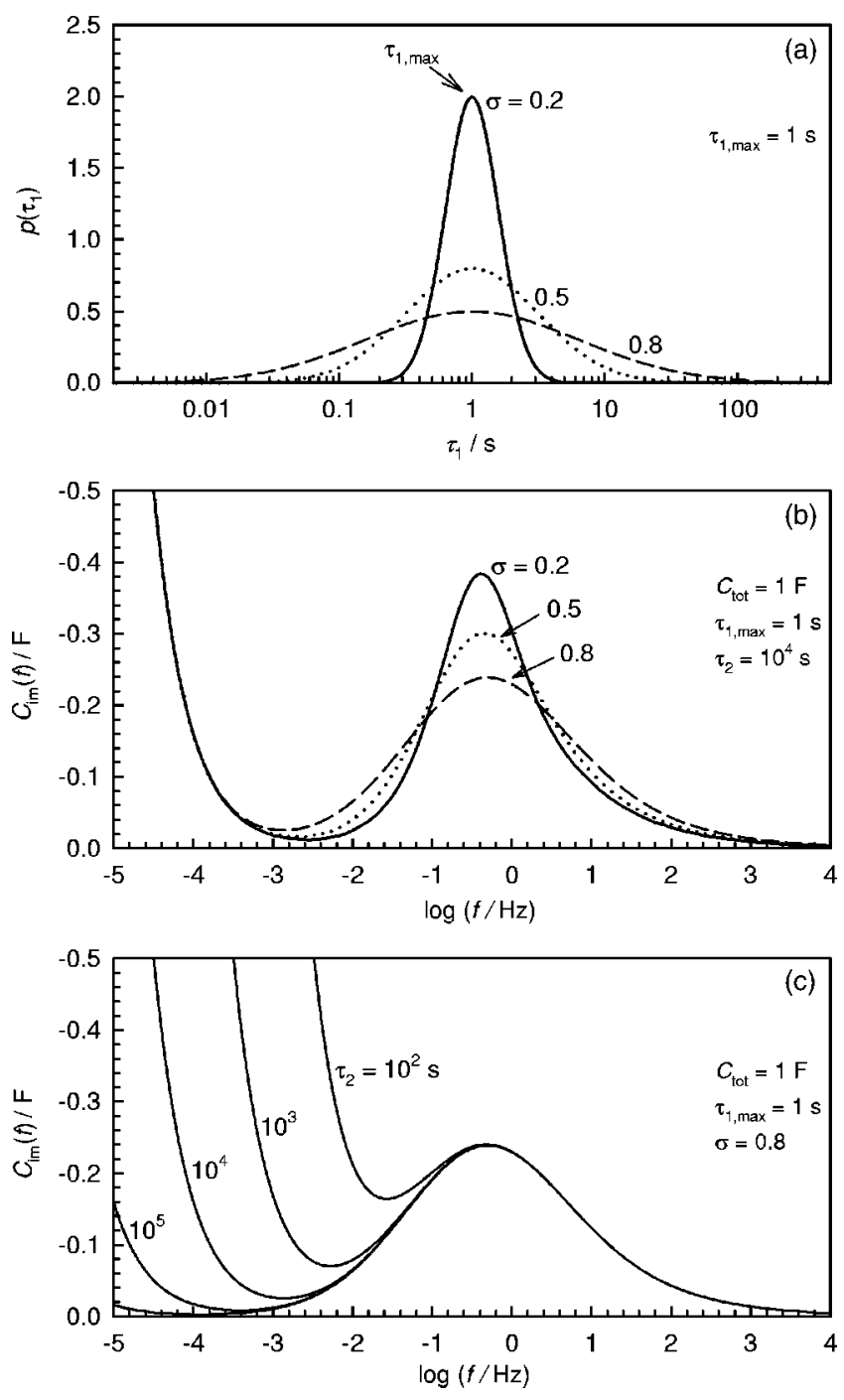

Figure 3. (a) Log-normal $\tau_{1}$ distribution as a function of standard deviation $(\sigma)$; (b) the simulated imaginary capacitance plots with a variation in $\sigma$; and (c) the simulated imaginary capacitance profiles as a function of $\tau_{2}$ while the other variables are being fixed.

$$
p\left(\tau_{1}\right)=\frac{d\left[C\left(\tau_{1}\right) / C_{\mathrm{tot}}\right]}{d \log \tau_{1}}=\frac{1}{\sqrt{2 \pi} \sigma} \exp \left[-\frac{1}{2 \sigma^{2}}\left(\log \tau_{1}-\log \tau_{1, \max }\right)^{2}\right]
$$

In this work, $p\left(\tau_{1}\right)$ is assumed to be a log-normal function based on the fact that pore size measured by nitrogen gas adsorption technique is commonly log-normally distributed. Here, $p\left(\tau_{1}\right)$ is defined by the maximum $\tau_{1}\left(\tau_{1, \max }\right)$ and standard deviation $(\sigma)$ that reflects the degree of dispersion in $\tau_{1}$ (Fig. 3a). ${ }^{10-12,22}$ As $\sigma$ increases, while $C_{\text {tot }}$ and $\tau_{1 \text { max }}$ in Eq. 13 are fixed, the capacitive peak becomes lower and broader as simulated in Fig. 3b, but the resistive tail at lower frequency region is not altered. The peak area and peak frequency are proportional to the total capacitance $\left(C_{\text {tot }}\right)$ and the inverse of maximum $\tau_{1}\left(\tau_{1, \max }\right)$ as indicated in Eq. 14

$$
A=0.682 C_{\text {tot }}, \quad f_{\mathrm{p}}=\frac{0.404}{\beta^{2}} \frac{1}{\tau_{1, \max }}
$$

Here, $\beta$ is the empirical parameter that varies according to the broadness in $\tau_{1}$ distribution, which approaches unity as the distribution becomes narrower. Note that Eq. 14 is identical to Eq. 6 when $\beta=1$, which is pertinent to the single-pore system. Figure $3 \mathrm{c}$ shows

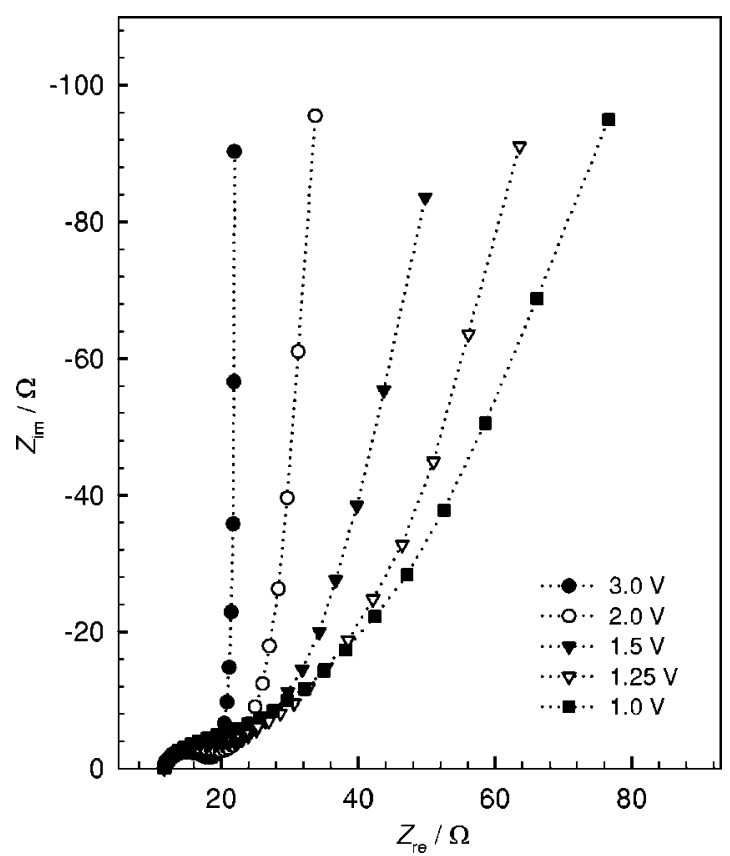

Figure 4. Nyquist plots taken with the BP electrode as a function of applied dc potential. The used electrolyte was $1.0 \mathrm{M} \mathrm{Et}_{4} \mathrm{NBF}_{4}$ dissolved in propylene carbonate. Note that the deviation from verticality in the low-frequency region becomes severe at more negative potentials.

the simulated imaginary capacitance profiles using Eq. 11-13, where $\tau_{2}$ is varied while the other variables are fixed. As $\tau_{2}$ decreases, the resistive tail shifts to the higher frequency direction, similarly to the single-pore system. The extent of overlapping between the capacitive peak and resistive tail is determined by the relative value of $\tau_{2} / \tau_{1, \max }$ and broadness of the capacitive peak.

\section{Experimental}

The EDLC performance, including the leakage current behavior, of a porous carbon called BP (Kuraray Chemical Co., Japan) was examined using the complex capacitance analysis. The surface area of BP measured by the Brunauer, Emmett, and Teller (BET) method was $1748 \mathrm{~m}^{2} \mathrm{~g}^{-1}$ and the average pore diameter was $2.1 \mathrm{~nm} .{ }^{23}$ For the preparation of composite electrode, the mixture of BP, Ketjenblack ECP-600JD (as an additive for conductivity enhancement), and sodium carboxymethylcellulose (as a binder, Aldrich Chem.) (75:20:5 in weight ratio) was coated on an etched aluminum foil (as a current collector, apparent area $=1 \mathrm{~cm}^{2}$ ) by the slurry coating method. A three-electrode configuration was used in the ac impedance measurement, where the same carbon composite electrode (apparent area $=18 \mathrm{~cm}^{2}$ ) and Li foil were used as the counter and reference electrode, respectively. The electrolytes used in the experiments were $1.0 \mathrm{M}$ of quaternary ammonium tetrafluoroborates dissolved in propylene carbonate (Tomiyama Chemical Co.). The ac impedance measurements were made over the frequency range of $10^{6}-5 \times 10^{-3} \mathrm{~Hz}$ with ac amplitude of $10 \mathrm{mV}$ (Zahner, im6e), while the dc potential was gradually changed from 3.0 to $1.0 \mathrm{~V}$ (vs. $\mathrm{Li} / \mathrm{Li}^{+}$). To precondition the BP electrode, the potential was repeatedly cycled between 3.0 and $1.0 \mathrm{~V}$ and maintained at each dc potential for an additional $2 \mathrm{~h}$ before the measurement. To evaluate the potential-dependent leakage behavior, the BP electrode was polarized at each dc potential and the potential decay was monitored as a function of time at the open-circuit condition. The experimental ac impedance data were processed and analyzed with Mathcad 2000 Professional (MathSoft, Inc.) software.

\section{Results and Discussion}

Figure 4 shows the Nyquist plot for the BP electrode that was 


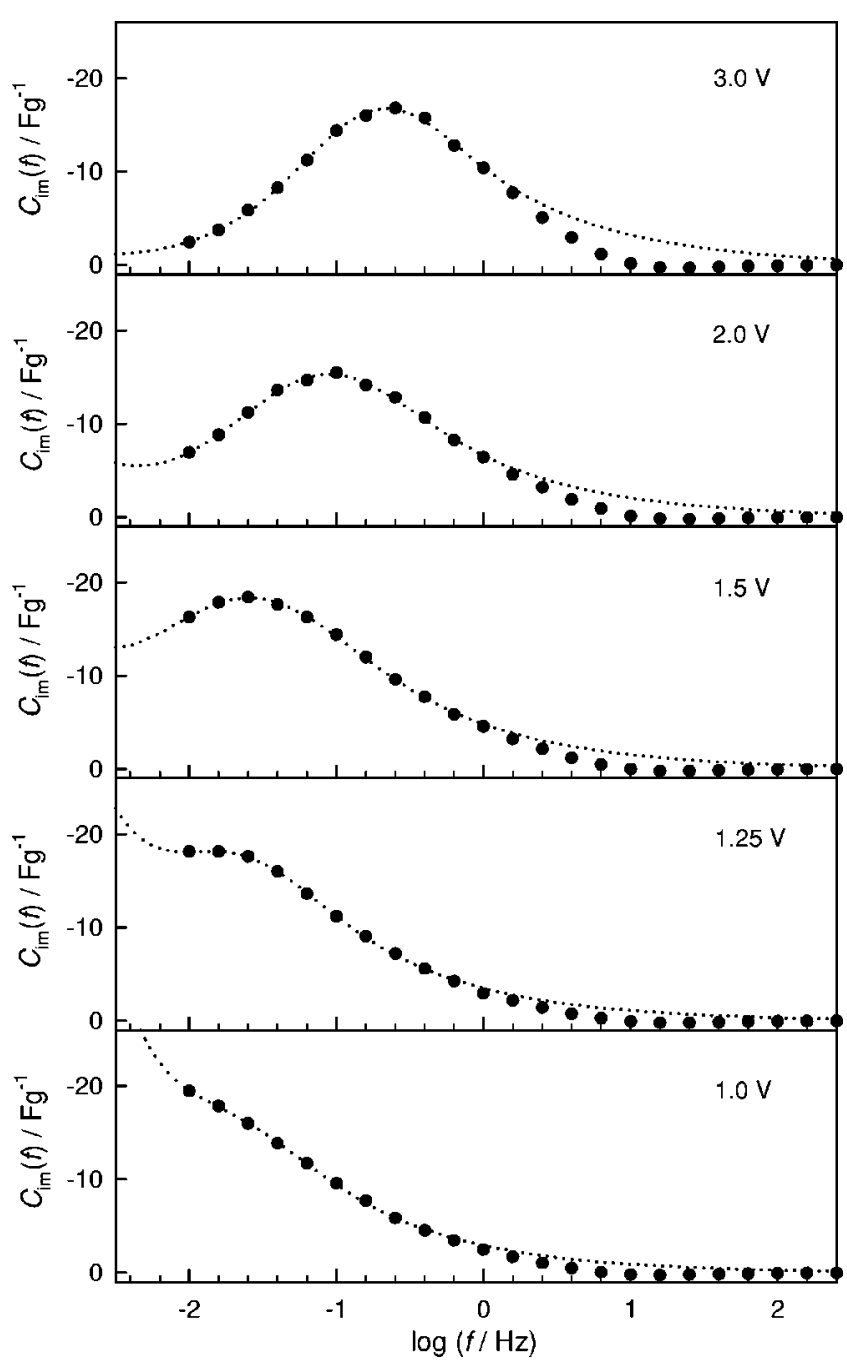

Figure 5. The imaginary capacitance profiles taken with the BP electrode as a function of applied dc potential. The electrolyte used was $1.0 \mathrm{M} \mathrm{Et}_{4} \mathrm{NBF}_{4}$ dissolved in propylene carbonate. The experimental data are indicated as the closed circles and the best-fitted ones as the dotted lines. Note the evolution of a resistive tail in the low-frequency region and the shift of capacitive peak to the lower frequency direction with negative polarization.

derived from the impedance data obtained at different dc potentials. The near-to-vertical line appearing in the low-frequency region in the $3.0 \mathrm{~V}$ plot suggests that the nonuniformity in pore geometry is not significant in this carbon. Considering that the measurement was made with the identical electrode, and the pore structure of carbon is not greatly affected by the polarization, it is concluded that the everincreasing deviation from verticality at more negative potentials is associated with leakage current.

The impedance data were converted to the complex capacitance using Eq. 15, and the imaginary part is displayed as a function of $\log f$ in Fig. 5. In the data processing, the high-frequency data that are related to the bulk electrolyte resistance and contact resistance were removed before the conversion

$$
C^{*}(f)=\frac{1}{j 2 \pi f Z^{*}(f)}=-\frac{Z^{\prime \prime}(f)}{2 \pi f\left|Z^{*}(f)\right|^{2}}-j \frac{Z^{\prime}(f)}{2 \pi f\left|Z^{*}(f)\right|^{2}}
$$

A typical capacitive peak is observed without resistive tail at the rest potential $\left(3.0 \mathrm{~V} v s . \mathrm{Li} / \mathrm{Li}^{+}\right)$, indicative of a negligible leakage current at this potential. As the potential moves to the negative direction, however, the resistive tail grows and the capacitive peak moves to the lower frequency direction, leading to a gradual overlap between two components. It is found by visual inspection that $(i)$ the

\begin{tabular}{|c|c|c|c|c|}
\hline $\begin{array}{c}E \\
\left(\mathrm{~V} \text { vs. } \mathrm{Li} / \mathrm{Li}^{+}\right)\end{array}$ & $C_{\text {tot }}\left(\mathrm{F} / \mathrm{g}^{-1}\right)$ & $\sigma$ & $\log \left(\tau_{1, \max } / \mathrm{s}\right)$ & $\log \left(\tau_{2} / \mathrm{s}\right)$ \\
\hline 1.0 & $48 \pm 5$ & $0.39 \pm 0.08$ & $1.4 \pm 0.1$ & $1.9 \pm 0.2$ \\
\hline 1.25 & $51 \pm 3$ & $0.37 \pm 0.05$ & $1.31 \pm 0.07$ & $2.2 \pm 0.2$ \\
\hline 1.5 & $59 \pm 2$ & $0.48 \pm 0.03$ & $1.21 \pm 0.04$ & $2.7 \pm 0.4$ \\
\hline 2.0 & $46.1 \pm 0.5$ & $0.38 \pm 0.02$ & $0.68 \pm 0.01$ & $2.8 \pm 0.2$ \\
\hline 3.0 & $47.2 \pm 0.3$ & $0.30 \pm 0.01$ & $0.28 \pm 0.01$ & $3.8 \pm 0.8$ \\
\hline
\end{tabular}

${ }^{a}$ The used electrolyte was $1.0 \mathrm{M} \mathrm{Et}_{4} \mathrm{NBF}_{4}$ dissolved in propylene carbonate.

total capacitance is not largely changed (from the peak area); (ii) the rate capability becomes poorer (from the shift of peak frequency to the lower frequency direction); and (iii) the leakage current increases (from the resistive tail) as the potential moves to the negative direction. The ready prediction on the EDLC parameters from the imaginary capacitance plots is the strong point of this method over the conventional Nyquist analysis.

The more accurate and quantitative EDLC parameters can be obtained by a nonlinear curve fitting with Eq. 11 to the experimental imaginary capacitances. The imaginary capacitance profiles that are best-fitted to the experimental data are indicated as dotted lines in Fig. 5. The best-fitted parameters for total capacitance $\left(C_{\text {tot }}\right)$, standard deviation $(\sigma)$, and two time constants $\left(\tau_{1, \max }\right.$ and $\left.\tau_{2}\right)$ are listed in Table I. Several features are immediately apparent in Fig. 5 and Table I. First, the simulated values are in good agreement with the experimental data below $1 \mathrm{~Hz}$, but a discrepancy appears at the high-frequency region that is caused by an incomplete removal of the high-frequency data in the data processing period. Second, $\tau_{2}$ decreases gradually as the potential becomes more negative, indicative of a smaller charge-transfer resistance for leakage reactions at more negative potentials (the last column in Table I). The increase of leakage current with cathodic polarization was further ascertained by the self-discharge experiment. As seen in Fig. 6, the selfdischarge reactions are negligible when the BP electrode is polarized at $2.0 \mathrm{~V}$. If the self-discharge reactions were measurable, the potential would decay from $2.0 \mathrm{~V}$ to the rest potential (ca. $3.0 \mathrm{~V} v$ s. $\mathrm{Li} / \mathrm{Li}^{+}$ in this system). When the polarization voltage moves to the negative direction, however, the potential decay become more significant,

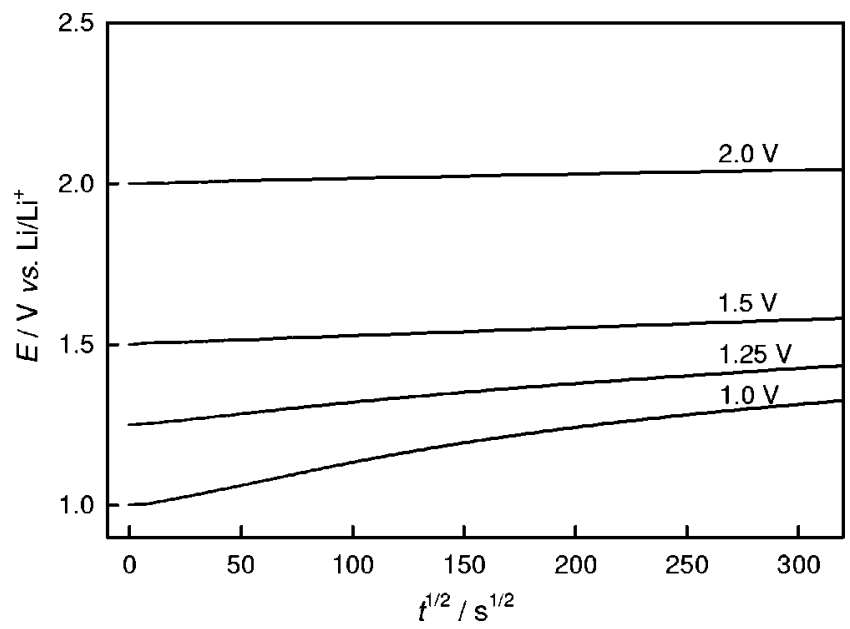

Figure 6. The potential decay curves traced at the open-circuit condition as a function of the charging potential. The electrolyte used was $1.0 \mathrm{M} \mathrm{Et}_{4} \mathrm{NBF}_{4}$ dissolved in propylene carbonate. Note that the decay from the charging potential to the rest potential $\left(3.0 \mathrm{~V} v s\right.$. $\left.\mathrm{Li} / \mathrm{Li}^{+}\right)$becomes more significant at more cathodic polarization, indicative of a larger leakage current. 


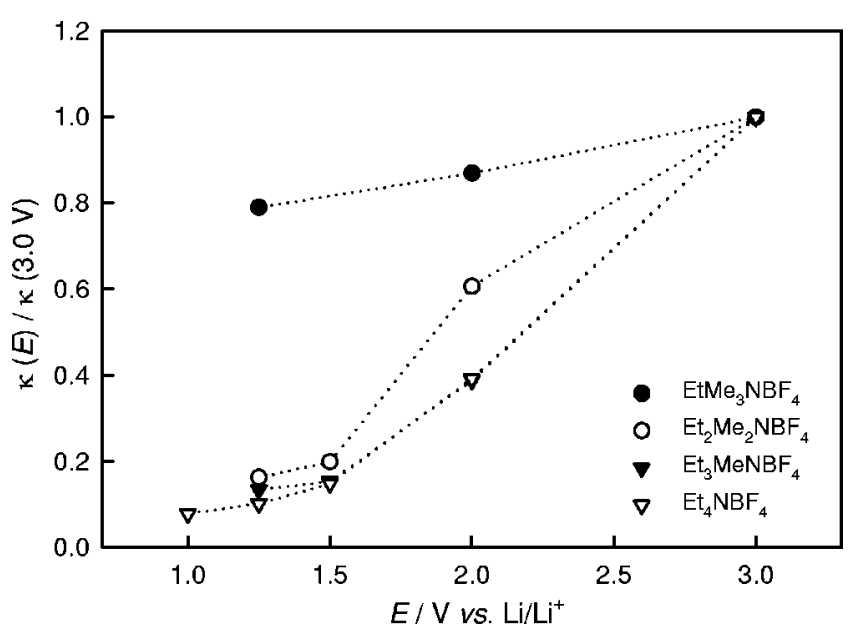

Figure 7. The potential-dependent ionic conductivity in pores that is normalized by the value at $3.0 \mathrm{~V}\left(v s\right.$. $\left.\mathrm{Li} / \mathrm{Li}^{+}\right)$. The data were obtained in the electrolytes prepared with four different quaternary ammonium salts dissolved in propylene carbonate $(1.0 \mathrm{M})$, where $\mathrm{Et}=$ ethyl and $\mathrm{Me}=$ methyl.

indicative of a larger leakage current. The leakage current appearing at the cathodic potentials may be associated with the electrochemical reduction of electrolyte, impurities, or surface functional groups on this carbon. Among these, the last possibility can be discarded because it is very likely that the surface active groups are partially or wholly removed when the imposed potential is more negative than the rest potential.

The third observation in Fig. 5 is the gradual shift of capacitive peak to the lower frequency direction at more negative potentials. As Eq. 14 reads, the $\tau_{1, \max }$ values in Table I become larger as the potential moves to the negative direction. This implies that the rate capability of BP electrode becomes poorer as the capacitor charging time becomes longer with an increase in $\tau_{1}$ value. This feature is likely caused by a decrease in the ionic conductivity inside pores at more negative potentials because the parameters related to the pore geometry $(L$ and $r)$ in Eq. 5 are invariant against electrode potential. To ascertain this premise, the following relation was derived from Eq. 5

$$
\kappa(E) \propto \frac{C_{\text {tot }}(E)}{\tau_{1, \max }(E)}
$$

The potential-dependent ionic conductivity in pores $[\kappa(E)]$ that was estimated from the $C_{\text {tot }}(E)$ and $\tau_{1, \max }(E)$ values in Table I is presented in Fig. 7. The data were obtained with four different electrolyte solutions. At more negative potentials, the ionic conductivity in pores gradually decreases regardless of the quaternary ammonium salt used. Several experimental and theoretical studies suggested that ionic conductivity in pores is lower than bulk ion conductivity because of electrostatic interaction between ions and pore surface. ${ }^{24,25}$ In this particular system, it is very possible that the pore surface is negatively charged upon a negative polarization and has a stronger attraction for the quaternary ammonium ions. This would explain why the ionic conductivity and rate capability is lower at 1.0 $\mathrm{V}$ than at $3.0 \mathrm{~V}\left(\right.$ vs. $\left.\mathrm{Li} / \mathrm{Li}^{+}\right)$. The extent of conductivity decrease is in the order of $\mathrm{Et}_{4} \mathrm{~N}^{+}$(ion radius: $\left.0.343 \mathrm{~nm}\right)>\mathrm{Et}_{3} \mathrm{MeN}^{+}(0.327$ $\mathrm{nm})>\mathrm{Et}_{2} \mathrm{Me}_{2} \mathrm{~N}^{+}(0.313 \mathrm{~nm})>\mathrm{EtMe}_{3} \mathrm{~N}^{+}(0.298 \mathrm{~nm}),{ }^{26}$ which is consistent with the fact that ion-pore-surface interaction is higher with larger ions.

\section{Conclusion}

The leakage current behavior of porous carbon EDLC electrode was examined using the complex capacitance analysis. It was found that the complex capacitance analysis provides several advantageous features over the Nyquist analysis: $(i)$ The leakage current behavior appears as a resistive tail at lower frequency region than that for the capacitive peak. The appearance of tail and peak in different frequency regions allows us to estimate the EDLC parameters without the impedance data at the extremely low frequencies. (ii) The extent of leakage current can be estimated from the intensity of resistive tail. (iii) The total capacitance and rate capability of EDLC electrode can be estimated from the integrated area and position of capacitive peak, respectively. The more accurate and quantitative analysis is possible by a nonlinear curve fitting. A limitation in the quantitative analysis may, however, be found when either the leakage current is significant or the pore geometry (radius and length) has a wide distribution because the resistive tail and capacitive peak are severely overlapped.

The complex capacitance analysis was made on the practical EDLC electrode. The leakage current becomes intensified when the porous carbon electrode (BP) is negatively polarized. Also, the rate capability of this electrode becomes poorer with cathodic polarization, which is caused by a decrease in the ionic conductivity in pores.

\section{Acknowledgment}

This work was supported by KOSEF through the Research Center for Energy Conversion and Storage and also by the Division of Advanced Batteries in NGE Program (project no. 10016446).

\section{References}

1. M. Ishikawa, M. Morita, M. Ihara, and Y. Matsuda, J. Electrochem. Soc, 141, 1730 (1994).

2. M. Ishikawa, M. Ihara, M. Morita, and Y. Matsuda, Electrochim. Acta, 40, 2217 (1995).

3. B. E. Conway, W. G. Pell, and T.-C. Liu, J. Power Sources, 65, 53 (1997)

4. K. Xu, S. P. Ding, and T. R. Jow, J. Electrochem. Soc., 146, 4172 (1999).

5. B. W. Ricketts and C. Ton-That, J. Power Sources, 89, 64 (2000).

6. A. Lasia, J. Electroanal. Chem., 397, 27 (1995).

7. R. K. Shervedani and A. Lasia, J. Electrochem. Soc., 144, 2652 (1997)

8. C. Hitz and A. Lasia, J. Electroanal. Chem., 500, 213 (2001).

9. J.-P. Candy, P. Foulloux, M. Keddam, and H. Takenouti, Electrochim. Acta, 27, 1585 (1982).

10. H.-K. Song, Y.-H. Jung, K.-H. Lee, and L. H. Dao, Electrochim. Acta, 44, 3513 (1999).

11. H.-K. Song, H.-Y. Hwang, K.-H. Lee, and L. H. Dao, Electrochim. Acta, 45, 2241 (2000).

12. G.-J. Lee, S.-I. Pyun, and C.-H. Kim, J. Solid State Electrochem., 8, 110 (2004).

13. R. de Levie, in Advances in Electrochemistry and Electrochemical Engineering, P. Delahay, Editor, Vol. VI, p. 329, John Wiley \& Sons, New York (1967).

14. H. Keiser, K. D. Beccu, and M. A. Gutjahr, Electrochim. Acta, 21, 539 (1976).

15. J.-P. Candy, P. Foulloux, M. Keddam, and H. Takenouti, Electrochim. Acta, 26 , 1029 (1981).

16. W. G. Pell, B. E. Conway, W. A. Adams, and J. de Oliveira, J. Power Sources, 80, 134 (1999)

17. R. Kötz and M. Carlen, Electrochim. Acta, 45, 2483 (2000).

18. W. G. Pell and B. E. Conway, J. Electroanal. Chem., 500, 121 (2001).

19. K. Honda, T. N. Rao, D. A. Tryk, A. Fujishima, M. Watanabe, K. Yasui, and H. Masuda, J. Electrochem. Soc., 148, A668 (2001).

20. B. E. Conway and W. G. Pell, J. Power Sources, 105, 169 (2002).

21. C.-H. Kim, S.-I. Pyun, and H.-C. Shin, J. Electrochem. Soc., 149, A93 (2002)

22. J. H. Jang and S. M. Oh, J. Electrochem. Soc., 151, A571 (2004).

23. Kuraray Technical Reports, Kuraray Chemical Co.

24. J. Koresh and A. Soffer, J. Electrochem. Soc., 124, 1379 (1977).

25. W. Y. Lo, K. Y. Chan, M. Lee, and K. L. Mok, J. Electroanal. Chem., 450, 265 (1998).

26. M. Ue, J. Electrochem. Soc., 141, 3336 (1994). 